\title{
Perspectives of Electricity Storage in Polymer Capacitors
}

\author{
Duško Dudić*
}

\begin{abstract}
Department of Radiation Chemistry and Physics, "VINČA" Institute of Nuclear Sciences - National Institute of the Republic of Serbia, University of Belgrade, P.O. Box 522, 11001 Belgrade, Serbia
\end{abstract}

\begin{abstract}
The price and environmental aspects of electricity storage significantly affect the application of green technologies. The electrochemical batteries are currently the best choice for storing electricity for most industrial needs and products. Polymer capacitors show very low energy density compared to conventional batteries and therefore cannot be widely used for electricity disposal. At the same time, all other features of polymer capacitors that characterize battery systems are ideal. After a brief comparison of the basic properties of electrochemical and physical batteries, this paper presents the influence of electron trapping on the energy density of a polyethylene capacitor. The presented results indicate that the phenomenon of electron trapping in polymers can increase the energy deposit of polymer capacitors.
\end{abstract}

Keywords: Polymer, capacitor, electron trapping, energy density.

\section{INTRODUCTION}

Electricity storage is a crucial component of a large number of industrial products. The problem of electricity storage is a significant limiting factor for the use of autonomous electric vehicles and the application of electricity from solar panels. The success of the application of green technologies depends on the ability of scientists and engineers to develop sufficiently cheap and environmentally friendly rechargeable batteries. Due to their high energy capacity, electrochemical batteries are in intensive use today. Rechargeable lithium batteries are currently the best choice for storing electricity for most industrial needs and products. The favorable characteristics of these batteries are a large specific energy capacity (about $150 \mathrm{Wh} / \mathrm{kg}$ ) and a weak memory effect [1-3]. Environmental and safety risks associated with this type of battery, their price and a limited lifespan are the reasons why great efforts are being made in the search for a more suitable type of battery $[3,4]$. The development of physical batteries in which electricity is deposited without electrochemical processes is one of the perspectives. The absence of chemical processes would dramatically extend the life of such batteries compared to the electrochemical batteries, and the choice of materials for their manufacture could be more environmentally friendly. The polymer capacitor is a typical example of a physical battery. Compared to the rechargeable lithium batteries, the polymer capacitors show very low energy density $(\sim 0.1 \mathrm{Wh} / \mathrm{kg})$ and

*Address correspondence to this author at the Department of Radiation Chemistry and Physics, "VINČA" Institute of Nuclear Sciences - National Institute of the Republic of Serbia, University of Belgrade, P.O. Box 522, 11001 Belgrade, Serbia; E-mail: ddudic@outlook.com significantly higher power density [5-7]. Due to the mentioned characteristics, the polymer capacitors are used primarily in low-energy electronics, they can also be easily designed for high-voltage applications. Unless a significant increase in the energy density of the polymer capacitors is achieved, they will not find application in the massive storage of electrical energy.

The energy density of a polymer capacitor with a certain geometry is proportional to the applied voltage (electric field - quadratic dependence) and the permittivity (polarity) of the polymer used. The voltage that can be applied to the polymer capacitor is limited by the voltage of the polymer breakdown. Increasing the polarity of a polymeric material by blending or adding fillers is usually accompanied by a decrease in the dielectric strength of such a material relative to the pure polymer, and this topic is common in studies dealing with the storage of electricity in polymeric materials $[8,9]$. Linear polymers show lower dielectric permittivity compared to ceramics, but due to the possibility of applying very large electric fields, polymer capacitors generally show higher energy densities compared to ceramic capacitors [7]. The energy densities of polymer capacitors range from $1.1 \mathrm{~J} / \mathrm{cm}^{3}(\sim$ $0.05 \mathrm{Wh} / \mathrm{kg}$ ) - polypropylene (PP) capacitor to $11 \mathrm{~J} / \mathrm{cm}^{3}$ - polyvinylidene fluoride (PVDF) capacitor [5,7-9]. Polymer composites based on fluoropolymers and ceramic fillers have so far shown the highest energy densities, slightly exceeding the value of $30 \mathrm{~J} / \mathrm{cm}^{3}[7]$. The influence of charge trapping in electrostatic dielectric capacitors on their energy density has not been considered so far. This study shows that this effect is not negligible and that the processes of trapping and de-trapping of electrons in polymers can increase the energy deposit of the polymer capacitor. 
The possibility of increasing the energy deposit in the polymer capacitors is important because it could contribute to the wider use of these environmentallyfriendly batteries.

\section{EXPERIMENTAL}

The isotropic sheet of low-density polyethylene (LDPE), PE552 - HIP Pancevo Serbia ( $M w=110000$ $\left.\mathrm{g} \cdot \mathrm{mol}^{-1}\right)$, was made in a hot press at $460 \mathrm{~K}$ and pressure of $1.75 \mathrm{MPa}$ for $5 \mathrm{~min}$, followed by quenching in water at room temperature. The thickness of the sheet thus obtained was in the range of $0.5 \pm 0.05 \mathrm{~mm}$ and the calorimetric crystallinity was about $30 \%$. A square-shaped sample $(10 \mathrm{~cm} \times 10 \mathrm{~cm})$ were cut from the center of the sheet. One side of the obtained sample was gently treated with sandpaper (P900) and a graphite electrode (graphite spray) is applied to that side.

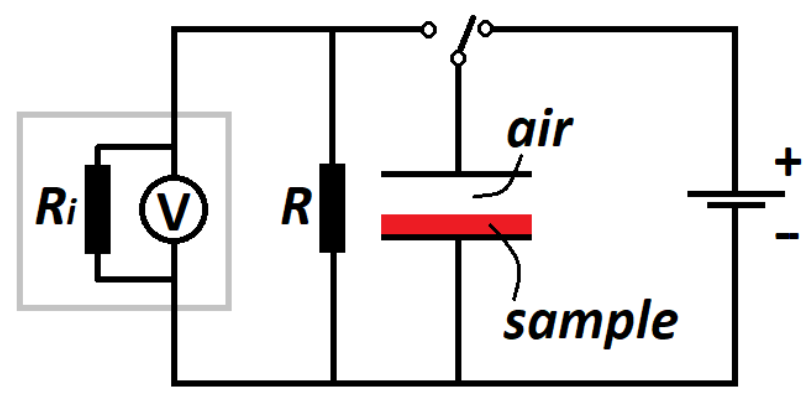

Figure 1: Experimental setup.

The electrical measurements were performed using a Keithley 2700 instrument and a DC voltage source. The LDPE sample was placed on the negative electrode of the capacitive cell (graphite-coated side), while it was separated from the positive electrode by air (1 $\mathrm{mm}$ air distance, the total distance between the electrodes was $1.5 \mathrm{~mm}$ ). Cell charging was performed using a voltage of $100 \mathrm{~V}$ (mean electric field in the sample is around $65 \mathrm{~V} / \mathrm{mm}$ ), charging times ranged from $3 \mathrm{~s}$ to $3000 \mathrm{~s}$. Discharge of the cell was performed via a parallel-connected resistor of $R=2.7 \mathrm{G} \Omega$ as shown in Figure 1. The instrument was used to measure the voltage on the cell over-discharging time, the time resolution was $0.2 \mathrm{~s}$. The discharge currents were obtained by dividing the measured voltage by the value of the known resistance $(2.7 \mathrm{G} \Omega$ ), taking into account the internal resistance of the instrument $\left(R_{i}=\right.$ $90 \mathrm{G} \Omega$ ).

\section{Rechargeable Batteries - Comparison}

The development of rechargeable batteries over the past 100 years has gone in the direction of increasing their energy density. The presence of heavy metals and their salts in electrochemical batteries is common. Due to the intensive growth of battery consumption, the environmental aspects of battery production and recycling are becoming imperative $[3,4,10]$. The battery life is also important, not only because of the price of the battery calculated by the year of use but also because of the environmental damage, which should also be calculated according to the period of use. The most important characteristics of the electrochemical and physical rechargeable batteries are presented in Table 1.

The price of batteries is not mentioned in Table 1 because it can vary significantly depending on the method of manufacture. Taking into account the other essential characteristics of the rechargeable batteries, listed in Table 1, only the polymer capacitor can be rated with three maximum ratings. The low energy density of the polymer capacitors prevents their wider application. Any improvement in the energy deposit of the polymer capacitors, which can be achieved without dirty technologies, brings this type of battery closer to the concept of an ideal battery.

Polyolefins, polymers composed only of carbon and hydrogen, are cheap and environmentally friendly materials for making batteries. Isotactic polypropylene (iPP) is a polyolefin that finds application in all branches of the industry due to its suitable thermomechanical, chemical and electrical (insulation) properties [11]. A more detailed insight into the economic aspects of the application of rechargeable batteries, on the example of an iPP/metal capacitor and the electrochemical batteries, is given in Table 2 .

The last row of Table 2 shows the prices of batteries with a capacity of $1 \mathrm{kWh}$, which are divided by the estimated time of use (iPP/metal 100 years and Li-bat. 10 years). Although the impact of the recycling price was not taken into account, the prices and weights shown in Table 2 clearly indicate the advantages of the electrochemical batteries over the polymer capacitors. The mentioned ratio, shown in Table 2 , can be improved in favor of the polymer capacitors only in the case of a significant improvement in their energy density. In doing so, all environmental aspects of that improvement must be acceptable. The study of the energy aspects of electron deposition in dielectrics could contribute to the improvement of the energy density of the capacitors. In the following chapter, on the example of a LPDE capacitor, it is shown that 
Table 1: Some Characteristics of Electrochemical and Physical Rechargeable Batteries $[2,6,7]$

\begin{tabular}{|c|c|c|c|c|c|}
\hline & Type & Energy density & Power density & Lifespan & Ecology \\
\hline \multirow{5}{*}{ 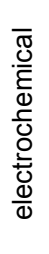 } & Metal-water/salt & medium & low & low & bad \\
\hline & Ni-metal (hydride) & medium & low & low & bad \\
\hline & Li-metal oxide & high & medium & medium & bad \\
\hline & Na-metal oxide & high & medium & medium & moderate \\
\hline & Supercapacitor & high & medium & medium & bad \\
\hline \multirow{2}{*}{ 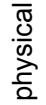 } & Ceramic capacitor & low & high & high & moderate \\
\hline & Polymer capacitor & low & high & high & the best \\
\hline
\end{tabular}

Table 2: Battery Economy: iPP/Metal Capacitor Versus Electrochemical Battery [2,7,12]

\begin{tabular}{|c|c|c|}
\hline & iPP/metal capacitor & electrochemical battery \\
\hline \hline Energy density [Wh/kg] & 0.05 & $10-150$ \\
\hline Power density [W/kg] & 250000 & 100 \\
\hline Lifespan [years] & 100 & 7 (Li-bat.) \\
\hline Battery weight required to deposit 1 kWh [kg] & 20000 & 50 -90\%, dirty technology \\
\hline Recycling & $100 \%$, cheap and clean technology & 31 (Li-bat.) \\
\hline Battery price [EUR/kg] & 3 & 22 EUR (Li-battery) \\
\hline $\begin{array}{c}\text { Disposal price of } 1 \mathrm{kWh} \text { calculated per year of } \\
\text { use without recycling [EUR] }\end{array}$ & 600 EUR & \\
\hline
\end{tabular}

electron trapping can increase the energy deposit in a polymer capacitor.

\section{LDPE Capacitor as an Electron Battery}

The nature and characteristics of electronic traps in linear polymers have been intensively studied in recent decades [13-15]. These studies provide a better understanding of the electric ageing of polymers. The electron trapping in a capacitor dielectric is treated as a harmful phenomenon because it is the cause of degradation processes in that dielectric [16]. Also, the delayed release of trapped charges from the dielectric causes technological problems with the high-capacity capacitors [17]. However, the possibility that trapped electrons can contribute to the energy density of capacitors has not been studied so far. This phenomenon is expected because the energy depth of the traps contributes to the screening of the Coulomb mutual repulsion of electrons and thus increases the charge deposit in the dielectric. The existence of trap energy could contribute to the discharge of trapped electrons through a process similar to the discharge of the electrochemical batteries. Discharging of one such physical battery would consist of rapidly discharging free charges, this is typical of a capacitor, and slowly discharging charges from the dielectric volume. Due to the existence of trap energy, especially if it is uniform, the release of electrons from the volume of the polymer could be characterized by a certain electromotive force. The discharge of one capacitor in which the trapping of electrons is expressed, the electronic battery, could be represented by Figure 2 .

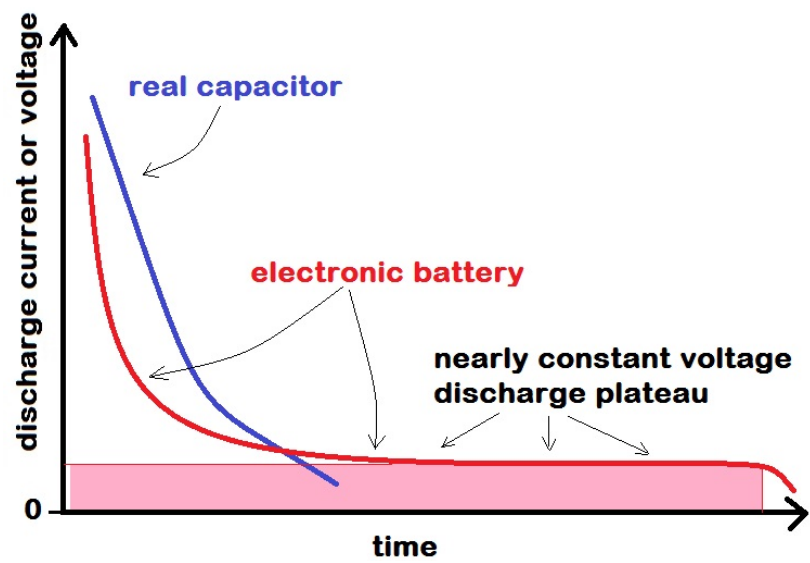

Figure 2: Discharge of a capacitor and an electronic battery.

Figure 3 shows the discharge currents of the tested LDPE capacitor. To avoid the influence of positive 
charge in the polymer, the LDPE film is leaned on the negative electrode and is separated by air from the positive electrode. The contribution of trapped electrons to discharge currents increases with increasing capacitor charging time.

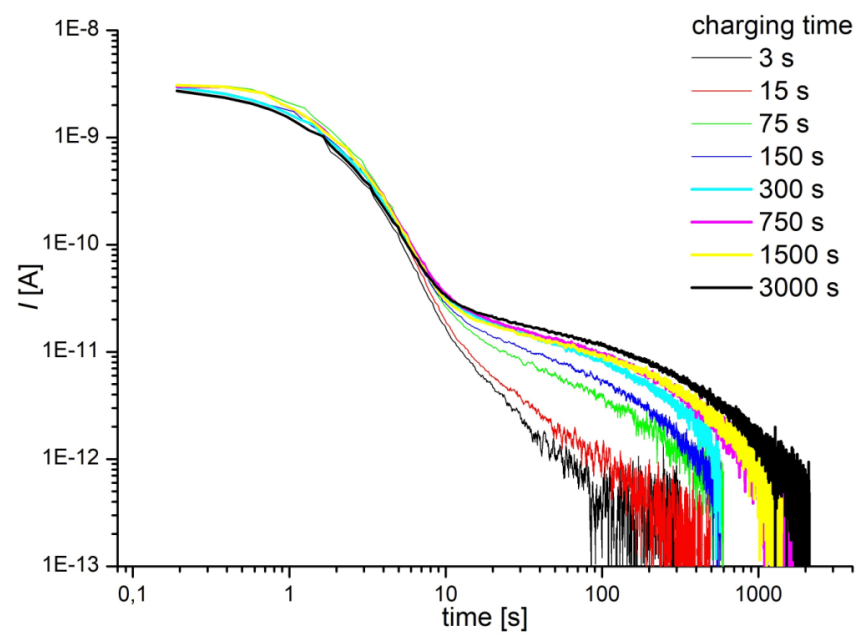

Figure 3: LDPE capacitor discharge currents, charging times are indicated in the figure.

The shape of the discharge current curve depends on the geometry of the capacitor, the charging electric field and the resistance over which the discharge is performed. Under the conditions described in the experimental section, the contribution of trapped electrons is observed from $10 \mathrm{~s}$ from the beginning of the discharge (Figure 3).

The energy released on the resistor over which the discharge was performed is shown in Figure $\mathbf{4 a}$, while the total charge released from the capacitor is shown in Figure $\mathbf{4 b}$. The aforementioned assumptions that
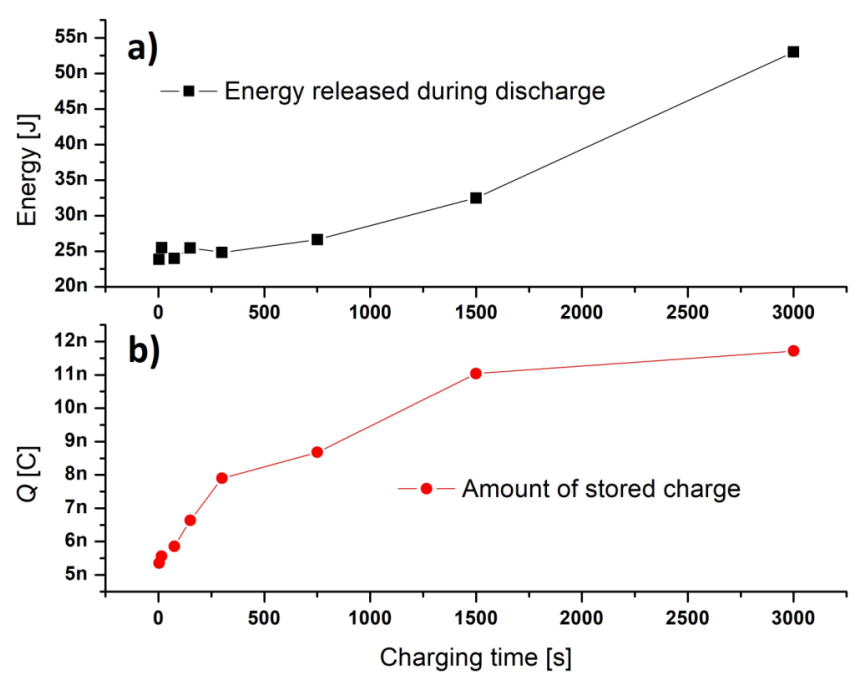

Figure 4: Total a) energy and b) charge released during the capacitor discharge depending on the charging time. electron trapping can increase the amount of deposited charge and energy in a polymer capacitor have been shown to be true for the case of the LDPE capacitor tested in this study. Figure $\mathbf{4}$ shows that the amounts of energy and charge released are approximately twice as high after charging the capacitor for $3000 \mathrm{~s}$ compared to $3 \mathrm{~s}$ charging time. The presented results suggest the need for further study of the influence of charge trapping on the energy density of polymer capacitors.

\section{CONCLUSION}

The price of depositing electricity in polymer capacitors is extremely high compared to the price of depositing electricity in electrochemical batteries, the reason for that is the very low energy density of polymer capacitors. At the same time, polymer capacitors show higher power density and significant environmental advantages. In this study, it was shown that the phenomenon of electron trapping increases the energy density of a LDPE-based capacitor. Given that LDPE is a non-polar polymer, it can be expected that the effect of electron trapping on energy density will be more pronounced in polar polymers and their composites. Studying the effects of charge trapping in dielectrics on their energy density could contribute to the wider use of capacitors for electrical storage. Depositing larger amounts of electricity in polymer capacitors would contribute to a cleaner planet.

\section{ACKNOWLEDGEMENTS}

This work was supported in part by the Ministry of Education, Science and Technological Development of the Republic of Serbia.

\section{REFERENCES}

[1] Tokimatsu K, Höök M, McLellan B, Wachtmeister H, Yasuoka $\mathrm{R}$, Nishio M. Energy modeling approach to the global energymineral nexus: Exploring metal requirements and the wellbelow $2{ }^{\circ} \mathrm{C}$ target with 100 percent renewable energy. Applied Energy 2018; 225: 1158-1175. https://doi.org/10.1016/j.apenergy.2018.05.047

[2] Ostergaard PA, Lund H, Stadler I. Towards $100 \%$ renewable energy systems. Applied Energy 2011; 88: 419-421. https://doi.org/10.1016/j.apenergy.2010.10.013

[3] Hannan MA, Lipu MSH, Hussain A, Mohamed A. A review of lithium-ion battery state of charge estimation and management system in electric vehicle applications: Challenges and recommendations. Renew Sust Energ Rev 2017; 78: 834-854. https://doi.org/10.1016/j.rser.2017.05.001

[4] Nitta N, Feixiang Wu, Lee JT, Yushin G. Li-ion battery materials: present and future. Materials Today 2015; 18: 252264.

https://doi.org/10.1016/j.mattod.2014.10.040

[5] Streibl M, Karmazin R. Materials and applications of polymer films for power capacitors with special respect to 
nanocomposites. IEEE Transactions on Dielectrics and Electrical Insulation 2018; 25: 2429. https://doi.org/10.1109/TDEl.2018.007392

[6] Zhang T, Guo M, Jiang J, Zhang X, Lin Y, Nan C-W, Shen Y. Modulating interfacial charge distribution and compatibility boosts high energy density and discharge efficiency of polymer nanocomposites. RSC Adv 2019; 9: 35990-35997. https://doi.org/10.1039/C9RA06933J

[7] Zhang H, Marwat MA, Xie B, Ashtar M, Liu K, Zhu Y, Zhang L, Fan P, Samart C, Ye Z-G. Polymer matrix nanocomposites with 1d ceramic nanofillers for energy storage capacitor applications. ACS Appl Mater Inter 2020; 12: 1-37. https://doi.org/10.1021/acsami.9b15005

[8] Liu $Y$, Hou $Y$, Ji $Q$ et al. Modulation of individual-layer properties results in excellent discharged energy density of sandwich-structured composite films. J Mater Sci Mater Electron 2020; 31: 7663-7671. https://doi.org/10.1007/s10854-020-03302-0

[9] Li JY, Zhang L, Ducharme S. Electric energy density of dielectric nanocomposites. Appl Phys Lett 2007; 90: 132901. https://doi.org/10.1063/1.2716847

[10] Nyholm L, Nyström G, Mihranyan A, Strømme M. Toward flexible polymer and paper-based energy storage devices. Adv Mater 2011; 23: 3751-3769. https://doi.org/10.1002/adma.201004134

[11] Škipina B, Petronijević IM, Luyt AS, Dojčinović BP, Duvenhage MM, Swart HC, Suljovrujić E, Dudić D. Ionic diffusion in iPP: DC electrical conductivity. Surfaces and Interfaces 2020; 21: 100772.

https://doi.org/10.1016/i.surfin.2020.100772
[12] Ahuja J, Dawson L,Lee R. A circular economy for electric vehicle batteries: driving the change. Journal of Property Planning and Environmental Law 2020; 12: 235-250. https://doi.org/10.1108/JPPEL-02-2020-0011

[13] Albrecht V, Janke A, Németh E, Spange S, Schubert G, Simon F. Some aspects of the polymers electrostatic charging effects. Journal of Electrostatics 2009; 67: 7-11. https://doi.org/10.1016/j.elstat.2008.10.002

[14] Dudić D, Škipina B, Dojčilović J, Novaković L, Kostoski D. Effects of charge trapping on the electrical conductivity of low-density polyethylene-carbon black composites. J App Pol Sci 2011; 121: 138-143. https://doi.org/10.1002/app.33421

[15] Zhou T-C, Chen G, Liao R, Xu Z. Charge trapping and detrapping in polymeric materials: Trapping parameters. Journal of Applied Physics 2011; 110: 043724. https://doi.org/10.1063/1.3626468

[16] Dissado LA, Thabet A. Simulation of electrical ageing in insulating polymers using a quantitative physical model. J Phys D: Appl Phys 2008; 41: 085412. https://doi.org/10.1088/0022-3727/41/8/085412

[17] Cho Y-M, Rhee J-H, Baek J-E, Ko K-C. Implementing a dielectric recovery strength measuring system for molded case circuit breakers. J Electr Eng Technol 2018; 13: 17521758.

http://doi.org/10.5370/JEET.2018.13.4.1752

https://doi.org/10.6000/1929-5995.2021.10.12

(C) 2021 Duško Dudić; Licensee Lifescience Global.

This is an open access article licensed under the terms of the Creative Commons Attribution License (http://creativecommons.org/licenses/by/4.0/) which permits unrestricted use, distribution and reproduction in any medium, provided the work is properly cited. 